\title{
Optimization of imaging conditions for composition determination by annular
}

\section{dark field STEM}

S. Firoozabadi*, P. Kükelhan*, T. Hepp, A. Beyer, K. Volz

${ }^{*}$ Both authors contributed equally

\section{Abstract}

Quantitative scanning transmission electron microscopy (STEM) allows composition determination for nanomaterials at an atomic scale. To improve the accuracy of the results obtained, optimized imaging parameters should be chosen for annular dark field imaging. In a simulation study, we investigate the influence of imaging parameters on the accuracy of the composition determination with the example of ternary III-V semiconductors. It is shown that inner and outer detector angles and semi-convergence angle can be optimized, also in dependence on specimen thickness. Both, a minimum sampling of the image and a minimum electron dose are required. These findings are applied experimentally by using a fast pixelated detector to allow free choice of detector angles.

Keywords: Quantitative STEM, Composition determination, four-dimensional STEM, Image simulation

\section{Introduction}

Quantitative scanning transmission electron microscopy (STEM) is a valuable tool for the characterization of nanostructures which is substantial for the development of new technologies. Aberration-corrected STEM allows a characterization at an atomic scale [1]. Particularly, annular dark field (ADF) imaging reveals information about the composition of a specimen [2]. Together with complementary image simulations 
composition determination on an absolute scale is possible $[3,4]$. The normalization of experimental data allows the direct comparison of experiment and simulation. Well-defined structures, like ternary III-V semiconductors have been and are used for method development. However, it should be noted that the conclusions drawn are valid also for other material systems.

Composition determination by ADF-STEM was applied to ternary III-V semiconductors early on $[5,6]$. Recently, the capability of single-electron accuracy for the composition determination of ternary III-V semiconductors was investigated [7]. The procedure proposed was then extended to cover both thickness and composition determination for ternary III-V semiconductors [8] and the composition of quaternary III-V semiconductors with two elements on each sub lattice [9] by taking into account the intensity of both sub lattices.

Being able to determine the composition of ternary III-V semiconductors with high accuracy and to assess this accuracy with simulation studies raises the question how accurate this composition determination can be. In particular, possible optimization and the influence of imaging conditions are of interest. Hence, several imaging parameters are analysed in this work. These include detector angles of the ADF detector, the probe semi-convergence angle, the electron dose used and the sampling of the image. All these parameters are evaluated in simulation studies for the ternary III-V semiconductors $\mathrm{Ga}(\mathrm{As}, \mathrm{Bi})$ and $(\mathrm{Ga}, \mathrm{In}) \mathrm{As}$. Eventually, the accuracy for typical experimental parameters can be estimated. Using the same methodology as presented here, the accuracy for composition determination can also be assessed for other materials. 
A huge improvement regarding the choice of detector angles is offered with the advent of fast pixelated detectors [10,11] resulting in four-dimensional STEM [12]. This allows a continuously variable choice of detector angles. Utilizing such a fast, pixelated electron detector, the optimized imaging conditions found in the simulation study are applied experimentally using aberration-corrected STEM.

\section{Image simulations}

STEM image simulations are performed with the software package STEMsalabim [13] which is an implementation of the multi-slice algorithm [14] optimized for highly parallelized computation. Here, different semi-convergence angles are chosen, which are 9.0, 15.1, 21.3 and $28.0 \mathrm{mrad}$. The values of 15.1 and $21.3 \mathrm{mrad}$ correspond to apertures that are present at the microscope at hand. For every semiconvergence angle, the optimum third-order spherical aberration $\mathrm{C}_{3}$ and defocus $\Delta f$ are chosen according to [15]. Based on [15], the value of defocus will be negligible. The corresponding defocus value calculated for $22 \mathrm{mrad}$ semi-convergence angle is less than $0.17 \mathrm{~nm}$ which is within the experimental accuracy of setting the defocus and will be regarded as zero for simplicity. The effect of chromatic aberration is taken into account by a defocus series of seven different defoci centred around the central defocus $\Delta f$ with a full-width half-maximum of $7.5 \mathrm{~nm}$ resulting from the chromatic aberration and the energy spread of the electrons in the microscope at hand [16]. For each defocus, 20 different atomic configurations are calculated for the frozen phonon approximation [17] to consider thermal diffuse scattering. The non-uniform detector sensitivity $[4,6]$ could be included by a detector scan $[6,18]$. However, for the simulation study where the detector angles are varied a perfect detector sensitivity is assumed and experimentally the response of the pixelated detector is considered by a gain map. The original sampling of the simulations in real space is 
40 pixels/nm. By $2 \mathrm{D}$ equivalent of the sinc-function interpolation this sampling can be adjusted afterwards [19]. The finite source size is accounted for by a Lorentzian convolution [20]. A finite electron dose can be considered by assuming shot noise with a width of $\sqrt{N}$ where $N$ is the number of electrons counted per pixel [21]. All microscope parameters used are given in Table 1.

\begin{tabular}{|l|l|}
\hline Electron energy & $200 \mathrm{kV}$ \\
\hline Semi-convergence angle & $9.0 ; 15.1 ; 21.3 ; 28.0 \mathrm{mrad}$ \\
\hline Astigmatism & $0 \mathrm{~nm}$ \\
\hline Defocus $\Delta f$ & $0 \mathrm{~nm}$ \\
\hline Third-order spherical aberration $\mathrm{C}_{3}$ & $-0.4 ;-1.0 ;-2.1 ;-3.6 \mu \mathrm{m}$ \\
\hline Fifth-order spherical aberration $\mathrm{C}_{5}$ & $5 \mathrm{~mm}$ \\
\hline Chromatic aberration $\mathrm{C}_{\mathrm{c}}$ & $1.5 \mathrm{~mm}$ \\
\hline$\Delta E$ & $1 \mathrm{eV}$ \\
\hline
\end{tabular}

Table 1: Microscope parameters used for STEM image simulations.

For each semi-convergence angle, eight $\mathrm{Ga}(\mathrm{As}, \mathrm{Bi})$ supercells with a size of $5 \times 5 \times$ 40 unit cells and a varying $\mathrm{Bi}$ concentration from $0-14 \%$ are simulated in [010]-direction. Additionally, eleven (Ga, In)As supercells with a size of $6 \times 6 \times 50$ unit cells and a varying In concentration from $0-50 \%$ are simulated in [010] orientation with a semi-convergence angle of $21.3 \mathrm{mrad}$. In each supercell, $\mathrm{Bi}$ or In atoms are randomly distributed in the GaAs matrix. Each slice for the multi-slice approach contains one atom per atomic column. Static atomic displacements are taken into account by a valence force field relaxation of Keating's potential [22].

For the detector angles chosen, an ADF-STEM image can be generated for which the Voronoi intensity of every atomic column [6] is used for a quantitative evaluation of the images.

\section{Experimental realization}


Composition determination of a sample containing GaAsBi quantum wells was performed by HAADF-STEM using the optimized parameters from the simulation study presented here. The investigated sample was grown via metalorganic vaporphase epitaxy (MOVPE) in an AIXTRON AIX 200 machine (Aixtron SE, Herzogenrath, Germany) under optimized conditions reported elsewhere [23].

Cross-sectional sample preparation is done in [010] orientation with a dual beam scanning electron microscope focused ion beam machine (JEOL JIB-4601F, JEOL Ltd., Tokyo, Japan). Milling voltages of the Ga ions are reduced from $30 \mathrm{kV}$ to $10 \mathrm{kV}$. Final specimen treatment is done with low voltage Ar ion milling of $900 \mathrm{eV}$ employing a NanoMill TEM specimen preparation system (model 1040, E. A. Fischione Instruments, Inc, Export, PA, United States) [24].

Experimental STEM data were acquired with a double aberration-corrected JEOL JEM 2200FS (JEOL Ltd., Tokyo, Japan). For image acquisition, a semi-convergence angle of $22 \mathrm{mrad}$ was used. The hexapoles of the imaging corrector were switched off to avoid distortion of the diffraction plane [25]. Detection of the convergent beam electron diffraction pattern at every $512 \times 512$ scan points was achieved with a pnCCD camera (pnDetector, Munich, Germany) [11] utilizing a dwell time of $500 \mu \mathrm{s}$. For every CBED, an offset map was subtracted, readout artefacts were corrected, and a gain correction was performed. This allows to apply a virtual ADF detector during the evaluation. Free control of the projector lens system was used to reach the desired diffraction angles. Normalization of resulting ADF images was intrinsically done by imaging the direct beam on the pnCCD camera.

Strain, in other words the Bi fraction, and thickness of the quantum wells were determined by modelling a HR X-ray diffractogram around the (004)-reflection. The 
diffractogram was recorded using the $\mathrm{Cu} \mathrm{K} \alpha$ line $(\lambda=1.5405 \AA)$ as $\mathrm{X}$-ray probe. Using the assumed zinc-blende GaBi lattice constant of $6.33 \AA \AA$ [26] a Bi concentration of $5.8 \% \mathrm{Bi}$ and a thickness of $7.2 \mathrm{~nm}$ were obtained. The X-ray diffractogram along with corresponding dynamical modelling shows a perfect agreement as can be seen in Fig. 1 .

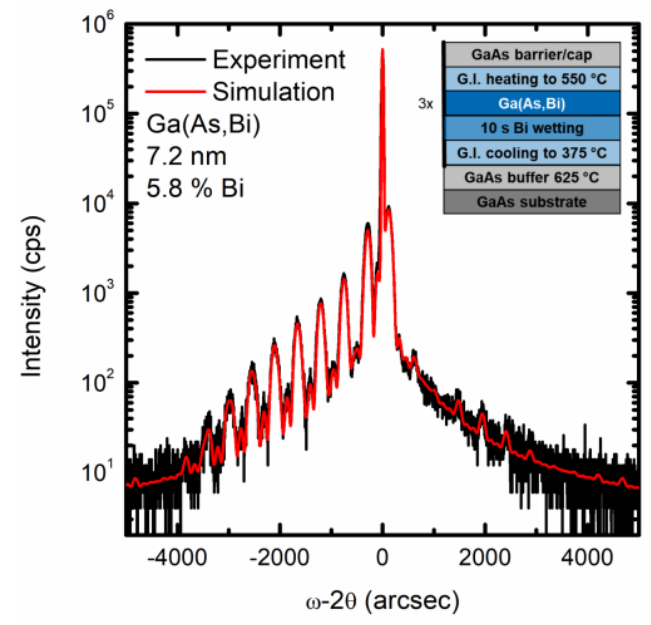

Fig 1: X-ray diffractogram and corresponding dynamical modelling of the investigated $\mathrm{Ga}(\mathrm{As}, \mathrm{Bi})$ sample. The inset sketches the growth sequence.

\section{Composition determination}

ADF-STEM images can be evaluated quantitatively to determine the composition of the investigated material. Here, the Voronoi intensity of every atomic column is analysed and related to its composition, i.e. its Bi content. From image simulations, an intensity composition relationship (see Fig. 2) can be ascertained and used for the composition determination for a given ADF-STEM image [7]. 


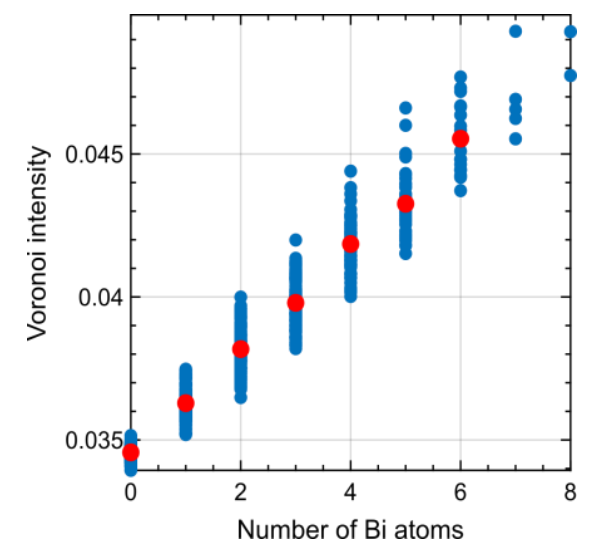

Fig. 2: Intensity composition relationship for $\mathrm{Ga}(\mathrm{As}, \mathrm{Bi})$ obtained at a thickness of 25 atoms per atomic column for a detector range of $70-280 \mathrm{mrad}$ with a semiconvergence angle of $21.3 \mathrm{mrad}$.

To obtain this intensity composition relationship, images are generated at a given thickness and for given microscope parameters for all simulated supercells. An infinite electron dose is assumed and the images are scaled up to 300 pixels $/ \mathrm{nm}$. Then, Voronoi intensity of every atomic column (blue data points) give a mean Voronoi intensity (red data points) for a given composition. This procedure is described and discussed in detail in [7]. For the given ADF-STEM image under investigation, Voronoi intensities are determined and then compared to the intensity composition relationship to find the composition of each atomic column. Here, a knowledge of the thickness of every atomic column is necessary and can be found from reference regions $[5,27]$ or using an iterative algorithm [8].

The composition evaluation described is statistically determined since one composition can result in different Voronoi intensities due to different z-height configurations of the impurity atoms within the TEM sample. This leads to a spread in Voronoi intensity and an overlap of Voronoi intensity for different compositions. Hence, the composition of an atomic column can only be determined correctly, i.e. with single atom accuracy, with a certain probability. As analysed before [7], one 
parameter that is very influential is the thickness of the atomic column, i.e. the total number of atoms of the atomic column.

In the following, the influence of detector angles, probe semi-convergence angle, electron dose and image sampling on the attainable accuracy is investigated.

\section{Influence of detector angles}

The influence of the detector angles on the attainable accuracy of the composition determination is investigated in simulation studies for $\mathrm{Ga}(\mathrm{As}, \mathrm{Bi})$ and $(\mathrm{Ga}, \mathrm{In}) \mathrm{As}$. In this detector angle study, a semi-convergence angle of $21.3 \mathrm{mrad}$ is employed and thicknesses of 10, 25and 40atoms per atomic columns are evaluated corresponding to $5.7,14.2$, and $22.8 \mathrm{~nm}$, respectively. The electron dose is assumed to be infinite and a real space sampling of 100 pixels $/ \mathrm{nm}$ is chosen for the image to evaluate. To solely analyse the composition determination, the thickness of every atomic column is assumed to be given, i.e. it is already found in a way mentioned above.

To investigate the accuracy of the composition determination, an ADF-STEM image is generated for the simulated supercell containing an average Bi concentration of $6 \%$ in the case of the material system $\mathrm{Ga}(\mathrm{As}, \mathrm{Bi})$ and an average In concentration of $20 \%$ in the case of the material system $(\mathrm{Ga}, \mathrm{In})$ As. Here, the accuracy of the composition determination depends on actual concentration as Fig. 2 suggests. For comparison, the analogous investigation for a simulated supercell containing an average concentration of $2 \%$ in material system $\mathrm{Ga}(\mathrm{As}, \mathrm{Bi})$ is depicted in Fig. $\mathrm{S} 1$ showing higher accuracy than $\mathrm{GaAs}_{0.94} \mathrm{Bi}_{0.06}$. This is done for every thickness at different detector angles. The inner detector angle is varied from $30-120 \mathrm{mrad}$ in steps of $10 \mathrm{mrad}$ while the detector range, i.e. outer-inner angle, is changed from $30-270 \mathrm{mrad}$ in steps of $10 \mathrm{mrad}$ but limited by a maximum outer detector angle of 
$300 \mathrm{mrad}$. For small inner angles there is a potential influence of amorphous layers and inelastic scattering $[28,29]$ which is not considered here. Hence, one might want to omit these small inner angles for an experimental application. Using the same parameters as for the image generation, an intensity composition relationship is determined. Then, the composition of every atomic column is determined. For the 121 group $\mathrm{V}$ atomic columns of $\mathrm{Ga}(\mathrm{As}, \mathrm{Bi})$ and the 121 group III atomic columns of (Ga,In)As evaluated in every image, one obtains a fraction of atomic columns for which the composition is determined correctly. This fraction is used as a measure for the accuracy of the composition determination. Worth noticing that even the columns which are not correctly determined are off by \pm 1 or \pm 2 atoms depending on the thickness according to [7]. In the case of 10 atoms thickness, the worngly assigned columns are off by a maximum of \pm 1 substitutional atoms with $0 \%$ (all atomic columns are estimated correctly) to $5.0 \%$ depending on the applied detection angles. At 25 atoms thickness, the percentage of wrongly determind atomic columns by \pm 1 atom and \pm 2 atoms deviation are $1.7-21.5 \%$ and $0-0.8 \%$, respectively. The numbers rise to $12.4-27.3 \%$ and $0-6.6 \%$ in the case of 40 atom thickness. These overestimation and underestimation in columns should be equal due to statistics, leading to an overall correct average composition.
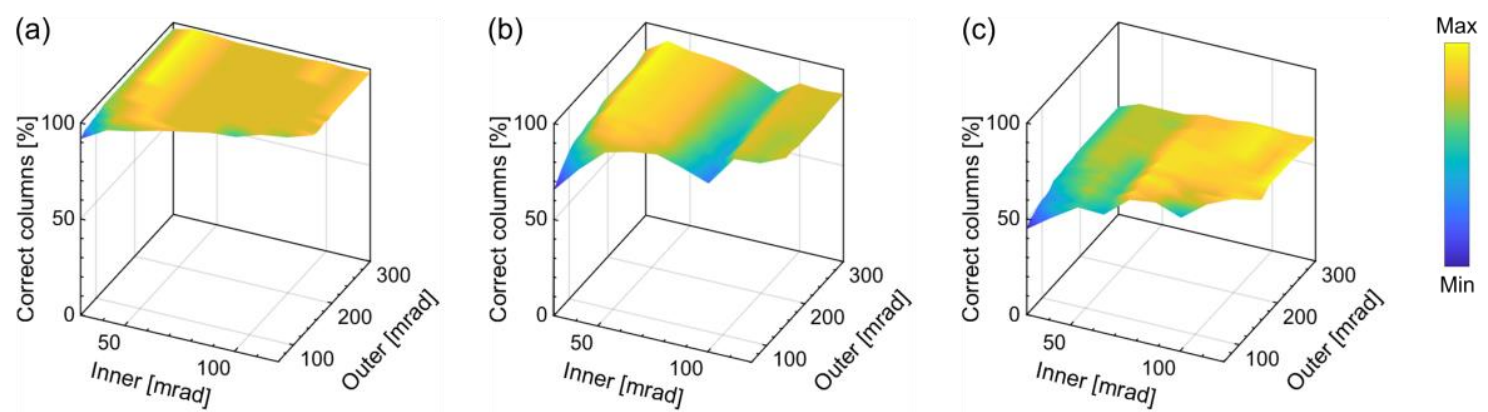

Fig. 3: Accuracy of composition determination for $\mathrm{GaAs}_{0.94 \mathrm{Bi}} \mathrm{B}_{06}$ depending on the inner and outer detector angle for a thickness of 10 (a), 25(b) and 40 (c) atoms. 
In Fig. 3, the percentage of correctly determined atomic columns for $\mathrm{Ga}(\mathrm{As}, \mathrm{Bi})$ is represented depending on the inner and outer detector angles for a thickness of 10 (a), 25(b) and 40 atoms per atomic column (c), respectively. As expected, with increasing thickness the general accuracy of the composition determination decreases [7]. Additionally, there clearly are preferable detector angles for each thickness, where the attainable accuracy for composition determination is better than for other detector angles. Here, the inner detector angle is more influential due to the higher scattering intensity being present but also the outer detector angle can be optimized.

For a thickness of 10 atoms, the highest accuracy of the composition determination is $99 \%$ and is achieved for an inner detector angle of $40 \mathrm{mrad}$ with an outer detector angle ranging from $180-300 \mathrm{mrad}$. The highest accuracy for a thickness of 25 atoms is $93 \%$ for an inner detector angle of $40 \mathrm{mrad}$ with outer detector angles of $230-300 \mathrm{mrad}$. For a thickness of 40 atoms, the highest attainable accuracy is $66 \%$ with an inner detector angle of $120 \mathrm{mrad}$ and outer detector angles of $180-$ $240 \mathrm{mrad}$. It is worthy to mention that the dips in the accuracy graphs at around 90 mrad inner detector angle in all thicknesses coincides the first order laue zone (FOLZ) of GaAs0.94 Bio.06. The coherent information related to FOLZ is in fact unsuitable for the quantification of composition using the naturally incoherent method of HAADF-STEM.

Hence, to obtain an optimized accuracy of the composition determination a careful choice of the detector angles is necessary. It is notable that the optimal detector angles do not necessarily coincide with the given geometry of the ADF detector at hand. Thus, the application of a fast pixelated detector to generate virtual ADFSTEM images with custom inner and outer detector angles becomes attractive. 
The optimal detector angles differ depending on the thickness. With increasing thickness, also the optimal inner detector angle increases which is analogous to atom counting. This effect is caused by the non-monotonic increase of the coherent contribution to the scattering cross section with thickness for low scattering angles [30].

Hence, for an optimization of the detector angles, first a knowledge of the thickness is necessary to choose the optimal detector angles for the thickness present in the actual specimen. This also becomes possible with 4D-STEM data and virtual ADFdetectors where all scattering angles are available.
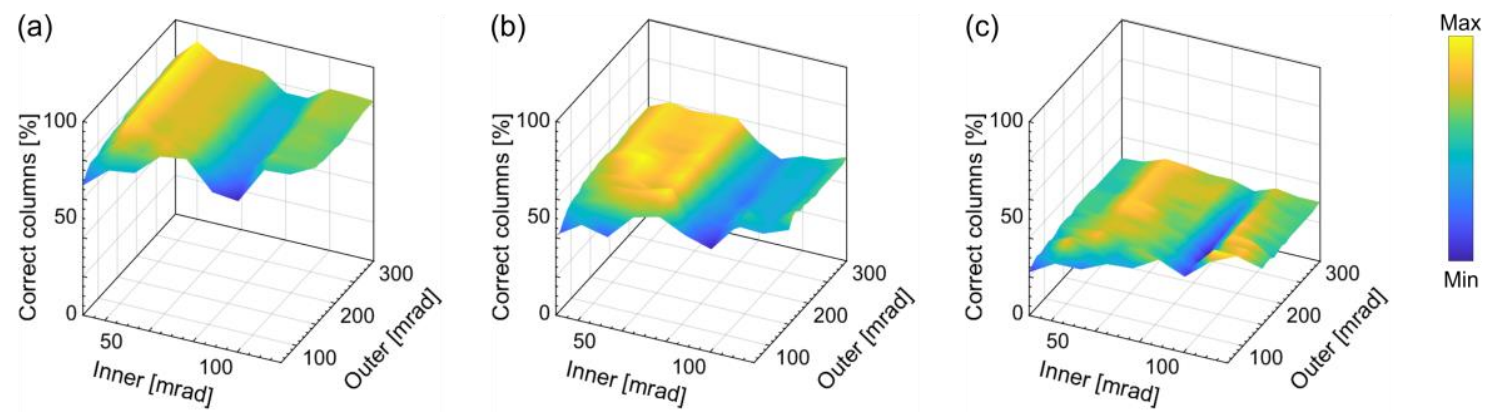

Fig. 4: Accuracy of composition determination for Ga0.8In0.2As depending on the inner and outer detector angle for a thickness of 10 (a), 25(b) and 40 (c) atoms. To stress the impact of different material systems on the optimal detector angles, also the results for $(\mathrm{Ga}, \mathrm{In})$ As are presented in Fig. 4. Here, the general trends described for $\mathrm{Ga}(\mathrm{As}, \mathrm{Bi})$ are the same. However, due to the smaller difference in atomic number between substitute atom and matrix atom the attainable accuracy is lower. Furthermore, the accuracy values of the optimum detector angles are different. For a thickness of 10 atoms, the highest attainable accuracy is $92 \%$ for an inner detector angle of $40 \mathrm{mrad}$ and outer detector angles of $220-300 \mathrm{mrad}$. The highest accuracy for a thickness of 25 atoms is $62 \%$ for an inner detector angle of $50 \mathrm{mrad}$ and outer detector angles of $170-180 \mathrm{mrad}$. For a thickness of 40 atoms, 
the highest accuracy of $36 \%$ can be achieved for an inner detector angle of $100 \mathrm{mrad}$ and an outer detector angle of $140 \mathrm{mrad}$. Interestingly, the accuracy is decreasing by increasing the inner detector angle higher than100 $\mathrm{mrad}$. As explained above, the dips in the accuracy graphs at around $90 \mathrm{mrad}$ coincide with the diffraction contrast corresponding with the first order laue zone (FOLZ) of Ga0.8Ino.2As which makes these angular ranges rather unsuitable for the quantification of composition.

\section{Influence of probe semi-convergence angle}

The influence of the semi-convergence angle on the attainable accuracy of the composition determination is investigated in simulation studies for $\mathrm{Ga}(\mathrm{As}, \mathrm{Bi})$ (see

Fig. 5) in the same manner as the influence of the detector angles. The same thicknesses of 10, 25 and 40 atoms per atomic column are used.

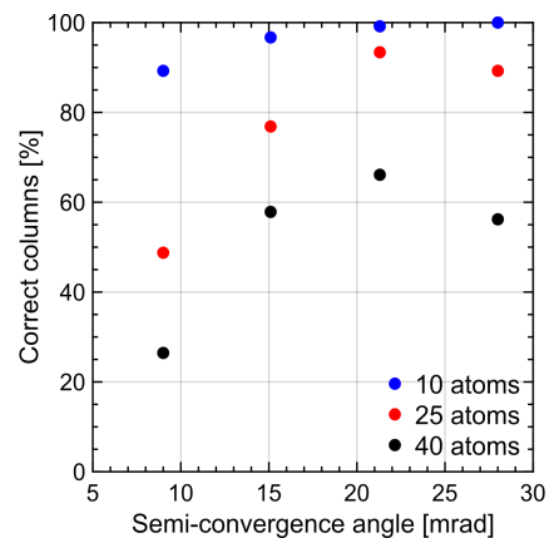

Fig. 5: Accuracy of composition determination for $\mathrm{GaAs}_{0.94 \mathrm{Bi}} .06$ depending on the semi-convergence angle for optimized detector angles. The electron dose is assumed to be infinite and the real space sampling of the image to evaluate is 100 pixels $/ \mathrm{nm}$.

The detector angles are optimized for each semi-convergence angle to give the highest accuracy attainable. For each semi-convergence angle, the highest accuracy is again obtained for the smallest thickness. For a thickness of 10 atoms, the 
attainable accuracy is increasing from $89 \%$ with a semi-convergence angle of $9.0 \mathrm{mrad}$ to $100 \%$ with a semi-convergence angle of $28.0 \mathrm{mrad}$. At a higher thickness of 25 atoms, the accuracy is rising from $49 \%$ at $9.0 \mathrm{mrad}$ to $93 \%$ at $21.3 \mathrm{mrad}$ but is decreasing again to $89 \%$ at $28.0 \mathrm{mrad}$. For a thickness of 40 atoms, this effect is more distinct as the accuracy is increasing from $26 \%$ at $9.0 \mathrm{mrad}$ to $66 \%$ at $21.3 \mathrm{mrad}$ and decreasing to $56 \%$ at $28 \mathrm{mrad}$. This dependence on the semi-convergence angle is caused by three detrimental effects. Firstly, if the geometric aberrations are sufficiently low, the attainable image resolution is improving with a higher semi-angle, resulting in a higher accuracy of composition determination. On the other hand, the reduced depth of focus coming with a higher semi-angle leads to a larger influence of the $z$ height of an impurity atom within the TEM sample. This leads to a higher variance of image intensity for a given composition of the column, i.e. increased "error bars" in Fig. 2, and accordingly to a higher error in composition determination. Interestingly, Peng et. al. reached a similar optimum semi-convergent angle on a Bloch wave calculations investigating the HAADF image formation for aberration-corrected STEM. They stated that increasing the illumination angle results in a higher contribution of the $1 \mathrm{~s}$ bound state to an optimum value, after which the contribution of non-1s states increases. This leads to a changeover from higher channeling and projection-dominated imaging to depth-dependent imaging which is not favorable in our method [31]. Additionally, chromatic aberration is limiting the information attainable for semi-convergence angles higher than $21.9 \mathrm{mrad}$ for given conditions of electron energy, energy width and chromatic aberration coefficient.

Hence, depending on the thickness of your specimen a suitable choice of the convergence angle for ADF-STEM is beneficial. This has to be chosen before the 
measurement based on the specimen thickness expected from sample preparation or different semi-convergence angles can be used for image acquisition to choose the optimized value afterwards.

\section{Influence of electron dose}

For the investigations above, an infinite electron dose to acquire the image for evaluation is assumed. However, experimentally the electron dose applied is finite and might also be limited by the stability of the material under investigation [32]. A finite electron dose leads to shot noise of the electron detector and hence limits the attainable accuracy of an ADF-based composition determination. To investigate the effect of the electron dose, the electron dose applied to the image for evaluation is varied from $10^{0}$ to $10^{7} e^{-} \AA^{-2}$. For each electron dose, 500 independent images were evaluated. For this study, $\mathrm{Ga}(\mathrm{As}, \mathrm{Bi})$ is evaluated at thicknesses of 10,25 and 40 atoms for detector angles of $70-280 \mathrm{mrad}$ and a semi-convergence angle of $21.3 \mathrm{mrad}$, i.e. the optimum conditions derived before. The sampling of the image to evaluate is 100 pixels $/ \mathrm{nm}$. The results are presented in Fig. 6 .

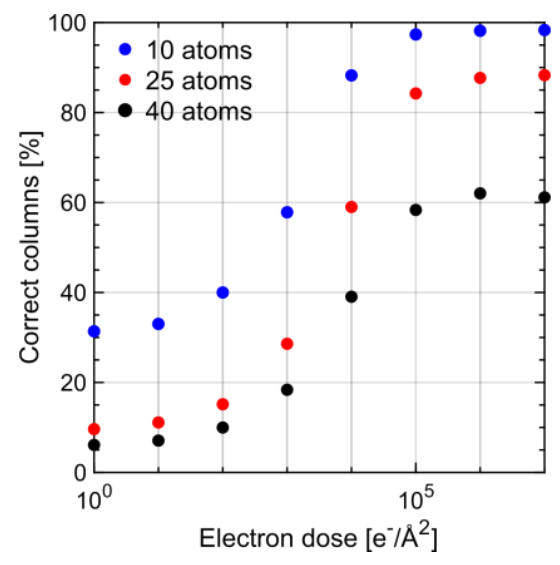

Fig. 6: Influence of electron dose on accuracy of composition determination for GaAs0.94Bio.06. The evaluation is performed with a semi-convergence angle of $21.3 \mathrm{mrad}$ for detector angles of $70-280 \mathrm{mrad}$. The sampling of the image to evaluate is 100 pixels $/ \mathrm{nm}$. 
For all thicknesses, there is a lower plateau for the accuracy for electron doses of 1 and $10 e^{-} \AA^{-2}$ for increasing electron doses, the accuracy increases as expected until it reaches a higher plateau for electron doses higher than $10^{5} e^{-} \AA^{-2}$. Hence, to reach the highest accuracy for composition determination electron doses of at least $10^{5} e^{-} \AA^{-2}$ is needed. Of course, also the sampling in interaction with the electron dose is influential on the accuracy but the general trend with two plateaus for the electron doses mentioned does not change for samplings of 50,150 and 200 pixels $/ \mathrm{nm}$ which are investigated in addition.

\section{Influence of sampling}

The sampling of the ADF image is deciding how accurately the image can be divided into Voronoi cells to yield representative Voronoi intensities. Experimentally, it is adjusted by the number of pixels of the image and the magnification. Hence, the influence of sampling is also investigated in a simulation study. Therefore, a simulated ADF image of $\mathrm{Ga}(\mathrm{As}, \mathrm{Bi})$ for detector angles of $70-280 \mathrm{mrad}$ and a semiconvergence angle of $21.3 \mathrm{mrad}$ is rescaled with samplings from 10 to 200 pixels $/ \mathrm{nm}$. An infinite electron dose is assumed. The accuracy obtained for the composition determination depending on the sampling is presented for thicknesses of 10,25 and 40 atoms in Fig. 7.

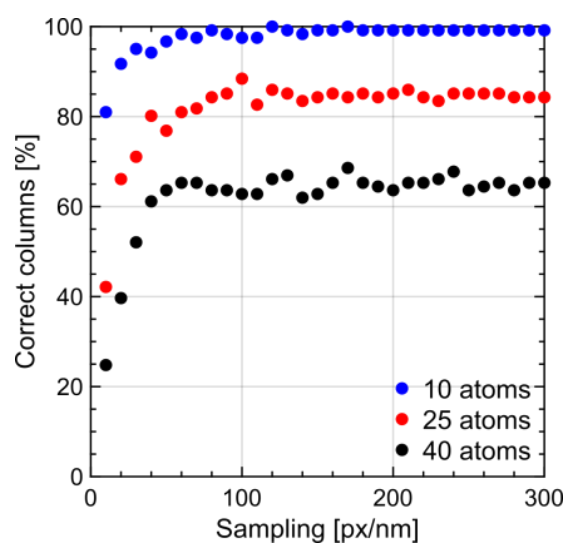




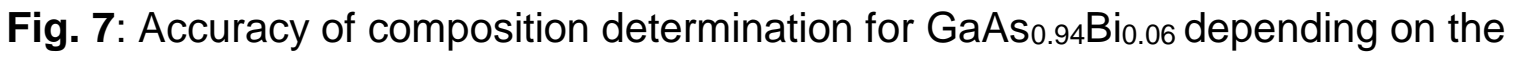
sampling of an ADF image with detector angles of 70-280 mrad and a semiconvergence angle of $21.3 \mathrm{mrad}$. An infinite electron dose is assumed.

For all thicknesses, the behaviour is similar. There is a strong increase in accuracy at small samplings that becomes more or less stable at roughly 80 pixels $/ \mathrm{nm}$. The accuracy above 80 pixels $/ \mathrm{nm}$ m only changes on a small scale probably caused by aliasing effects.

\section{Estimation of experimental accuracy}

After evaluating the influence of single parameters on the accuracy of composition determination, the accuracy reached for typical experimental imaging conditions without using the optimized parameters is assessed on the example of $\mathrm{Ga}(\mathrm{As}, \mathrm{Bi})$. These typical imaging conditions are summarized in Table 2.

\begin{tabular}{|l|l|}
\hline Specimen thickness & 25 atoms $(\sim 14.2 \mathrm{~nm})$ \\
\hline Semi-convergence angle & $21.3 \mathrm{mrad}$ \\
\hline Detector angles & $70-280 \mathrm{mrad}$ \\
\hline Sampling & 100 pixels $/ \mathrm{nm}$ \\
\hline Probe current & $40 \mathrm{pA}$ \\
\hline Number of images & 10 \\
\hline Dwell time & $3 \mu \mathrm{s}$ \\
\hline
\end{tabular}

Table 2: Typical experimental imaging conditions

Assuming that the thickness is already known, single-atom accuracy is reached for $50 \%$ of the atomic columns. The overall concentration of the supercell is overestimated by $0.5 \%$.

Since experimentally the thickness has to be determined also, this leads to another potential error in accuracy. To assess this, the thickness is determined by an iterative algorithm developed for the simultaneous determination of both specimen thickness and composition [8]. 


\section{Experimental results}

While specimen thickness and quality are a matter of sample preparation, semiconvergence angle, electron dose and sampling of the image can be adapted with a standard STEM setup. The experimental parameters of the used electron microscope are summarized in Table 3.

\begin{tabular}{|l|l|}
\hline Electron energy & $200 \mathrm{kV}$ \\
\hline Semi-convergence angle & $22 \mathrm{mrad}$ \\
\hline Astigmatism & $0 \mathrm{~nm}$ \\
\hline Defocus $\Delta f$ & $0 \mathrm{~nm}$ \\
\hline Third-order spherical aberration $\mathrm{C}_{3}$ & $-2.2 \mu \mathrm{m}$ \\
\hline Fifth-order spherical aberration $\mathrm{C}_{5}$ & $5 \mathrm{~mm}$ \\
\hline Chromatic aberration $\mathrm{C}_{\mathrm{c}}$ & $1.5 \mathrm{~mm}$ \\
\hline$\Delta E$ & $1 \mathrm{eV}$ \\
\hline
\end{tabular}

Table 3: Experimental parameters of the used electron microscope

A given ADF detector allows an adaption of the inner detector angle which is more influential but the ratio of inner and outer detector angle is normally fixed. To allow a free choice of both inner and outer detector angles, a fast, pixelated detector is useful.

The 4D-dataset shown here is acquired with $512 \times 512$ pixels squared which together with the magnification used yields a sampling of 45 pixels $/ \mathrm{nm}$. A positionaveraged CBED averaged over all real space pixels is shown in Fig. 8a. For the CBED of every real space pixel, a virtual ADF detector can be applied resulting in an ADF image. First, detector angles of $70-150 \mathrm{mrad}$ are used and the image is evaluated using an iterative determination of both thickness and composition [8] as both are unknown. From this first detector choice, an estimated average thickness of 48 atoms corresponds to $27.3 \mathrm{~nm}$ can be determined. As it is explained in Fig. $\mathbf{3}$, for this thickness there is an optimum detector range with highest accuracy. Here, a 
simulation study is performed for the corresponding thickness to determine the best detector range as $90-120 \mathrm{mrad}$.

The ADF image following from this optimization is shown in Fig. $\mathbf{8 b}$. The composition map following from applying the iterative algorithm is shown in Fig. 8c. Negative composition is allowed to consider noise.

By averaging this composition map perpendicular to the growth direction, concentration profiles can be obtained (see blue line in Fig. 8d). It is worth mentioning that the shaded areas depict the concentration standard deviation perpendicular to the growth direction. The width and concentration of the quantum well derived from high resolution X-ray diffraction (HR-XRD) (Fig. 1) is visualized as solid black line in Fig. 8d. There is a good agreement both in quantum well width and composition between the results obtained from HR-XRD and ADF. To emphasize the role of optimization, the concentration profile obtained by the typical detection angle of $70-150 \mathrm{mrad}$ is added as red line showing a distinct difference in respect to the HR-XRD as well as the concentration profile at the optimized detection angles. Hence, the successful application of the use of a fast, pixelated detector for quantitative ADF imaging with free choice of detector angles is shown. 
(a)

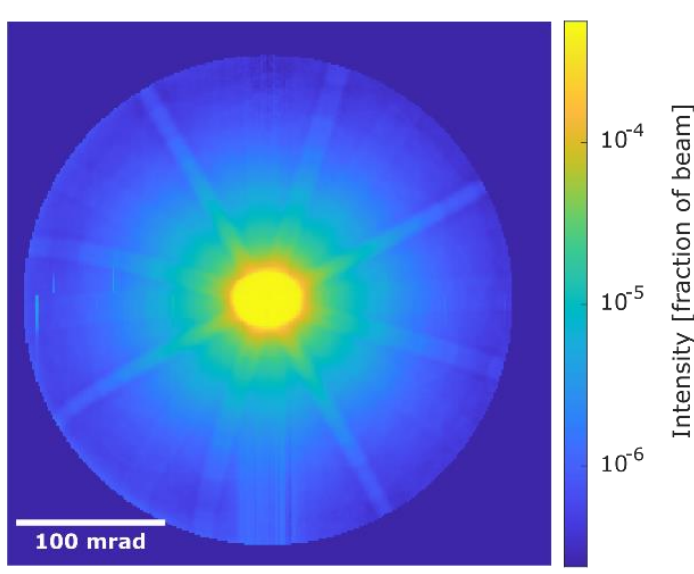

(c)

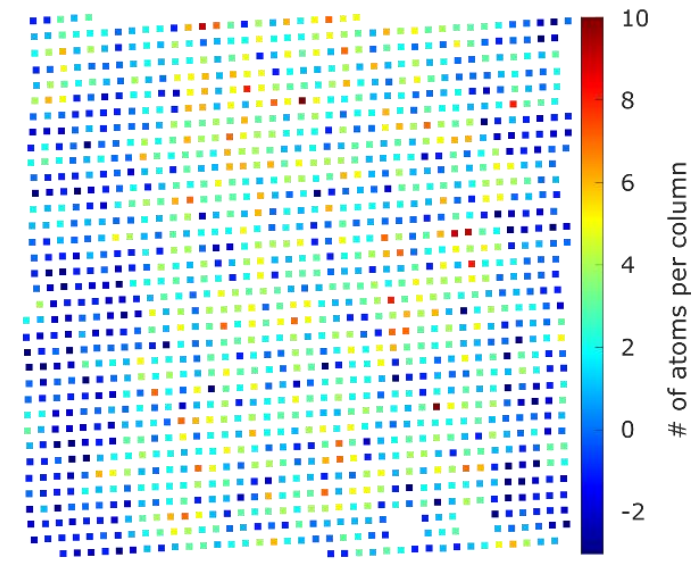

(b)
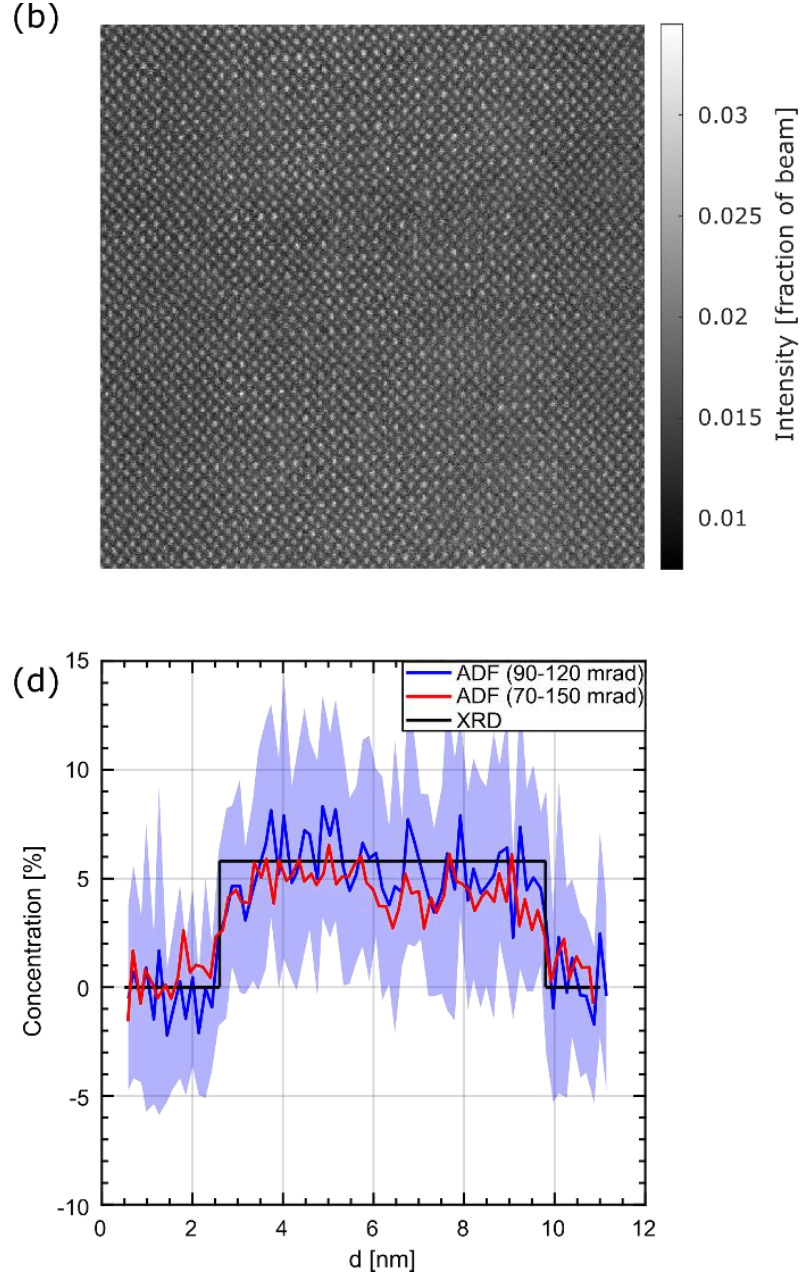

Figure 8: (a) Position-averaged CBED for experimental dataset. Using each CBED, a virtual ADF image can be obtained (b) which yields an atomic-resolution composition map. The concentration profile (d) following (c) is in good agreement with X-ray diffraction results.

\section{Summary}

In this work, it is shown that an optimization of imaging conditions for composition determination of ternary III-V semiconductors is possible and offers the potential to increase the accuracy. This requires a close interplay between experiment and simulation to find the best imaging conditions for every case, especially for different material systems and specimen thicknesses. By simulation studies, the influence of detector angles, semi-convergence angle, electron dose and sampling of the image is evaluated. Additionally, the accuracy of composition determination for typical 
experimental parameters is estimated. Considering these findings and using a fast, pixelated electron detector that allows free choice of detector angles, the outline procedure is applied experimentally to a $\mathrm{Ga}(\mathrm{As}, \mathrm{Bi})$ quantum well. The resulting geometry and composition of the sample is in a good agreement with HR-XRD. The use of a fast, pixelated electron detector for ADF imaging offers a huge potential for the future.

\section{Supplementary information}
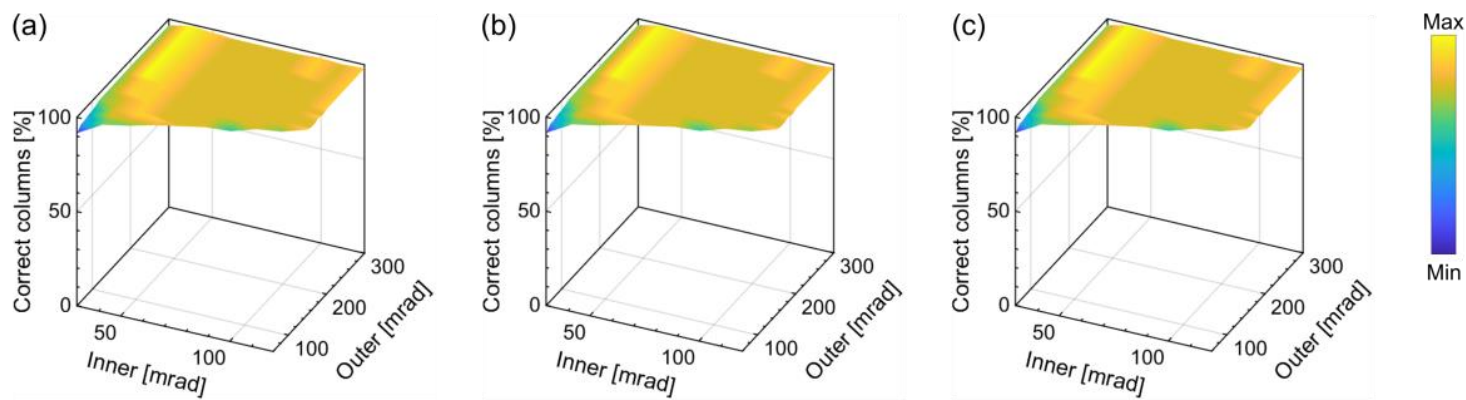

Fig. S1: Accuracy of composition determination for $\mathrm{GaAs}_{0.98} \mathrm{Bi}_{0.02}$ depending on the inner and outer detector angle for a thickness of 10 (a), 25(b) and 40 (c) atoms.

\section{Acknowledgements}

Financial support by the German Research Foundation (DFG) in the framework of SFB 1083 "Structure and Dynamics of Internal Interfaces" as well as by the BMBF in the framework of the "MehrSi project (03SF0525C) is greatly acknowledged. S. Firoozabadi acknowledges support of the EU in the framework of the project microSPIRE (ID: 766955). T. Hepp acknowledges financial support by the DFG in the framework of the research training group "Functionalization of Semiconductors" (GRK 1782).

\section{References}

[1] R. Erni, M.D. Rossell, C. Kisielowski, U. Dahmen, Atomic-Resolution Imaging with a Sub-50-pm Electron Probe, Phys. Rev. Lett. 102 (2009) 096101. https://doi.org/10.1103/PhysRevLett.102.096101. 
[2] S.J. Pennycook, L.A. Boatner, Chemically sensitive structure-imaging with a scanning transmission electron microscope, Nature. 336 (1988) 565-567.

[3] J.M. LeBeau, S.D. Findlay, L.J. Allen, S. Stemmer, Quantitative atomic resolution scanning transmission electron microscopy, Phys. Rev. Lett. 100 (2008) 206101. https://doi.org/10.1103/PhysRevLett.100.206101.

[4] J.M. LeBeau, S. Stemmer, Experimental quantification of annular dark-field images in scanning transmission electron microscopy, Ultramicroscopy. 108 (2008) 1653-1658. https://doi.org/10.1016/j.ultramic.2008.07.001.

[5] A. Rosenauer, K. Gries, K. Müller, A. Pretorius, M. Schowalter, A. Avramescu, K. Engl, S. Lutgen, Measurement of specimen thickness and composition in Alx Ga1 - x N / GaN using high-angle annular dark field images, Ultramicroscopy. 109 (2009) 1171-1182. https://doi.org/10.1016/j.ultramic.2009.05.003.

[6] A. Rosenauer, T. Mehrtens, K. Müller, K. Gries, M. Schowalter, P. Venkata Satyam, S. Bley, C. Tessarek, D. Hommel, K. Sebald, M. Seyfried, J. Gutowski, A. Avramescu, K. Engl, S. Lutgen, Composition mapping in InGaN by scanning transmission electron microscopy, Ultramicroscopy. 111 (2011) 1316-1327. https://doi.org/10.1016/j.ultramic.2011.04.009.

[7] L. Duschek, P. Kükelhan, A. Beyer, S. Firoozabadi, J.O. Oelerich, C. Fuchs, W. Stolz, A. Ballabio, G. Isella, K. Volz, Composition determination of semiconductor alloys towards atomic accuracy by HAADF-STEM, Ultramicroscopy. 200 (2019) 84-96. https://doi.org/10.1016/j.ultramic.2019.02.009.

[8] P. Kükelhan, A. Beyer, S. Firoozabadi, T. Hepp, K. Volz, Simultaneous 
determination of local thickness and composition for ternary III-V semiconductors by aberration-corrected STEM, Ultramicroscopy. 201 (2019). https://doi.org/10.1016/j.ultramic.2019.03.005.

[9] P. Kükelhan, A. Beyer, S. Firoozabadi, M. Sarkar, T. Hepp, K. Volz, Composition determination for quaternary III-V semiconductors by aberrationcorrected STEM, Manuscr. Prep. 206 (2019) 112814.

https://doi.org/10.1016/j.ultramic.2019.112814.

[10] D. Mcgrouther, M. Krajnak, I. Maclaren, D. Maneuski, V.O. Shea, D. Peter, Use of a hybrid silicon pixel ( Medipix ) detector as a STEM detector, 21 (2020) 1595-1596. https://doi.org/10.1017/S1431927615008752.

[11] H. Ryll, M. Simson, R. Hartmann, P. Holl, M. Huth, S. Ihle, Y. Kondo, P. Kotula, A. Liebel, K. Müller-Caspary, A. Rosenauer, R. Sagawa, J. Schmidt, H. Soltau, L. Strüder, A pnCCD-based, fast direct single electron imaging camera for TEM and STEM, J. Instrum. 11 (2016). https://doi.org/10.1088/17480221/11/04/P04006.

[12] C. Ophus, Four-Dimensional Scanning Transmission Electron Microscopy (4DSTEM): From Scanning Nanodiffraction to Ptychography and Beyond, Microsc. Microanal. (2019). https://doi.org/10.1017/S1431927619000497.

[13] J.O. Oelerich, L. Duschek, J. Belz, A. Beyer, S.D. Baranovskii, K. Volz, STEMsalabim: A high-performance computing cluster friendly code for scanning transmission electron microscopy image simulations of thin specimens, Ultramicroscopy. 177 (2017) 91-96.

https://doi.org/10.1016/j.ultramic.2017.03.010.

[14] E.J. Kirkland, Advanced computing in electron microscopy, Springer 
International Publishing, 2020.

[15] M. Haider, S. Uhlemann, J. Zach, Upper limits for the residual aberrations of a high-resolution aberration-corrected STEM, Ultramicroscopy. 81 (2000) 163175. https://doi.org/10.1016/S0304-3991(99)00194-1.

[16] K. Kuramochi, T. Yamazaki, Y. Kotaka, M. Ohtsuka, I. Hashimoto, K. Watanabe, Effect of chromatic aberration on atomic-resolved spherical aberration corrected STEM images, Ultramicroscopy. 110 (2009) 36-42. https://doi.org/10.1016/j.ultramic.2009.09.003.

[17] R.F. Loane, P. Xu, J. Silcox, Thermal vibrations in convergent-beam electron diffraction, Acta Crystallogr. Sect. A. 47 (1991) 267-278. https://doi.org/10.1107/S0108767391000375.

[18] S.D. Findlay, J.M. LeBeau, Detector non-uniformity in scanning transmission electron microscopy, Ultramicroscopy. 124 (2013) 52-60. https://doi.org/10.1016/j.ultramic.2012.09.001.

[19] C. Dwyer, Simulation of scanning transmission electron microscope images on desktop computers, Ultramicroscopy. 110 (2010) 195-198. https://doi.org/10.1016/j.ultramic.2009.11.009.

[20] A. Beyer, J. Belz, N. Knaub, K. Jandieri, K. Volz, Influence of spatial and temporal coherences on atomic resolution high angle annular dark field imaging, Ultramicroscopy. 169 (2016) 1-10. https://doi.org/10.1016/j.ultramic.2016.06.006.

[21] A. De Backer, K.H.W. van den Bos, W. Van den Broek, J. Sijbers, S. Van Aert, StatSTEM: An efficient approach for accurate and precise model-based 
quantification of atomic resolution electron microscopy images,

Ultramicroscopy. 171 (2016) 104-116.

https://doi.org/10.1016/j.ultramic.2016.08.018.

[22] P.N. Keating, Effect of invariance requirements on the elastic strain energy of crystals with application to the diamond structure, Phys. Rev. 145 (1966) 637645. https://doi.org/10.1103/PhysRev.145.637.

[23] P. Ludewig, Z.L. Bushell, L. Nattermann, N. Knaub, W. Stolz, K. Volz, Growth of $\mathrm{Ga}(\mathrm{AsBi})$ on $\mathrm{GaAs}$ by continuous flow MOVPE, J. Cryst. Growth. 396 (2014) 95-99. https://doi.org/10.1016/j.jcrysgro.2014.03.041.

[24] R.R. Cerchiara, P.E. Fischione, J. Liu, J.M. Matesa, A.C. Robins, H.L. Fraser, A. Genc, Raising the Standard of Specimen Preparation for AberrationCorrected TEM and STEM, Micros. Today. 19 (2011) 16-19. https://doi.org/10.1017/s1551929510001197.

[25] F.F. Krause, M. Schowalter, T. Grieb, K. Müller-Caspary, T. Mehrtens, A. Rosenauer, Effects of instrument imperfections on quantitative scanning transmission electron microscopy, Ultramicroscopy. 161 (2016) 146-160. https://doi.org/10.1016/j.ultramic.2015.10.026.

[26] S. Tixier, M. Adamcyk, T. Tiedje, S. Francoeur, A. Mascarenhas, P. Wei, F. Schiettekatte, Molecular beam epitaxy growth of Molecular beam epitaxy growth of GaAsBi, Appl. Phys. Lett. 82 (2013) 2245. https://doi.org/10.1063/1.1565499.

[27] G.T. Martinez, A. Rosenauer, A. De Backer, J. Verbeeck, S. Van Aert, Quantitative composition determination at the atomic level using model-based high-angle annular dark field scanning transmission electron microscopy, 
Ultramicroscopy. 137 (2014) 12-19.

https://doi.org/10.1016/j.ultramic.2013.11.001.

[28] T. Grieb, F.F. Krause, K. Müller-Caspary, S. Firoozabadi, C. Mahr, M.

Schowalter, A. Beyer, O. Oppermann, K. Volz, A. Rosenauer, Angle-resolved scanning transmission electron microscopy applied to Si: contributions, interpretation and sources of error, Submitted. (2020).

[29] A. Beyer, F.F. Krause, H.L. Robert, S. Firoozabadi, T. Grieb, P. Kükelhan, D. Heimes, M. Schowalter, K. Müller-Caspary, A. Rosenauer, K. Volz, Influence of plasmon excitations on atomic-resolution quantitative 4D scanning transmission electron microscopy, Sci. Rep. 10 (2020). https://doi.org/10.1038/s41598-020-74434-w.

[30] A. De Backer, A. De wael, J. Gonnissen, S. Van Aert, Optimal experimental design for nano-particle atom-counting from high-resolution STEM images, Ultramicroscopy. 151 (2015) 46-55.

https://doi.org/10.1016/j.ultramic.2014.10.015.

[31] Y. Peng, P.D. Nellist, S.J. Pennycook, HAADF-STEM imaging with subangstrom probes: A full Bloch wave analysis, J. Electron Microsc. (Tokyo). 53 (2004) 257-266. https://doi.org/10.1093/jmicro/53.3.257.

[32] S. Van Aert, A. De Backer, L. Jones, G.T. Martinez, B. Armand, Control of Knock-On Damage for 3D Atomic Scale Quantification of Nanostructures : Making Every Electron Count in Scanning Transmission Electron Microscopy, Phys. Rev. Lett. 122 (2019) 066101. https://doi.org/10.1103/PhysRevLett.122.066101. 